\title{
THE NAPOLEON-BARLOTTI THEOREM IN PENTAGONAL QUASIGROUPS
}

\author{
STIPE VIDAK \\ University of Zagreb, Croatia
}

\begin{abstract}
Pentagonal quasigroups are IM-quasigroups in which the additional identity $(a b \cdot a) b \cdot a=b$ holds. GS-quasigroups are IM-quasigroups in which the identity $a(a b \cdot c) \cdot c=b$ holds. The relation between these two subclasses of IM-quasigroups is studied. The geometric concepts of GS-trapezoid and affine regular pentagon, previously defined and studied in GS-quasigroups, are now defined in a general pentagonal quasigroup. Along with the concepts of the regular pentagon and the centre of the regular pentagon, previously defined in pentagonal quasigroups, this enables formulations and proofs of some theorems of the Euclidean plane in a general pentagonal quasigroup. Among these theorems is the famous Napoleon-Barlotti theorem in the case $n=5$.
\end{abstract}

\section{INTRODUCTION}

A quasigroup $(Q, \cdot)$ is a groupoid in which for given $a, b \in Q$ each of the equations $a \cdot x=b$ and $y \cdot a=b$ has the unique solution. A quasigroup $(Q, \cdot)$ is called IM-quasigroup if it satisfies the identities of idempotency and mediality:

$$
\begin{gathered}
a a=a, \\
a b \cdot c d=a c \cdot b d .
\end{gathered}
$$

The immediate consequences of these identities are the identities known as elasticity, left distributivity and right distributivity:

$$
\begin{aligned}
& a b \cdot a=a \cdot b a, \\
& a \cdot b c=a b \cdot a c,
\end{aligned}
$$

2010 Mathematics Subject Classification. 20N05.

Key words and phrases. IM-quasigroup, pentagonal quasigroup, regular pentagon, affine regular pentagon. 


$$
a b \cdot c=a c \cdot b c .
$$

Definition 1.1. Pentagonal quasigroup is an IM-quasigroup $(Q, \cdot)$ in which the identity of pentagonality holds:

$$
(a b \cdot a) b \cdot a=b .
$$

The previous definition can be found in [8]. In pentagonal quasigroups, along with pentagonality and the identities that hold in any IM-quasigroup, some other identities hold. They are stated in the next theorem proved in [8].

THEOREM 1.2. In every IM-quasigroup $(Q, \cdot)$ the identity (1.6) and the identities

$$
\begin{gathered}
(a b \cdot a) c \cdot a=b c \cdot b, \\
(a b \cdot a) a \cdot a=b a \cdot b, \\
a b \cdot(b a \cdot a) a=b
\end{gathered}
$$

are mutually equivalent and they imply the identity

$$
a(b \cdot(b a \cdot a) a) \cdot a=b
$$

for every $a, b, c \in Q$.

Example 1.3. The basic example of the pentagonal quasigroup is $C(q)=$ $(\mathbb{C}, *)$, where $*$ is defined by

$$
a * b=(1-q) a+q b
$$

and $q$ is a solution of the equation

$$
q^{4}-3 q^{3}+4 q^{2}-2 q+1=0 .
$$

This example motivates the introduction of many geometric concepts in pentagonal quasigroups. Actually, some concepts can be defined more generally in medial and IM-quasigroups and the whole idea of geometry of medial quasigroups and its subclasses comes from [10,12].

We can regard elements of the set $\mathbb{C}$ as points of the Euclidean plane. For any two different points $a, b \in \mathbb{C}$ the equality $a * b=(1-q) a+q b$ can be written in the form

$$
\frac{a * b-a}{b-a}=\frac{q-0}{1-0} .
$$

That means that the points $a, b$ and $a * b$ are vertices of a triangle directly similar to the triangle with vertices 0,1 and $q$. Each $q_{i}, i=1,2,3,4$ gives a certain type of triangle and we get so called characteristic triangles for pentagonal quasigroups (see [8]). In $C\left(q_{1}\right)$ the point $a * b$ is the third vertex of the regular pentagon determined by its adjacent vertices $a$ and $b$. Any identity in the pentagonal quasigroup $C(q)=(\mathbb{C}, *)$ can be interpreted as a theorem of the Euclidean geometry. Some illustrations can be found in [8]. 
In [9] basic geometry of pentagonal quasigroups was developed. In this paper the concepts of the regular pentagon, the regular decagon and the centre of the regular pentagon, all defined and studied in [9], will be of a particular interest.

DeFINITION 1.4. GS-quasigroup is an idempotent quasigroup $(Q, \cdot)$ in which the identity

$$
a(a b \cdot c) \cdot c=b
$$

holds.

ExAmPLE 1.5. The basic example of the GS-quasigroup is $C(q)=(\mathbb{C}, *)$, where $*$ is defined by

$$
a * b=(1-q) a+q b
$$

and $q$ is a solution of the equation

$$
q^{2}-q-1=0
$$

GS-quasigroups were defined and studied in [11] where the next theorem was proved.

THEOREM 1.6. In any GS-quasigroup $(Q, \cdot)$ the mediality holds.

It follows immediately that GS-quasigroups are actually IM-quasigroups in which the identity (1.12) holds.

In already mentioned article some other identities that hold in GS-quasigroups were studied and some basic geometric concepts in GS-quasigroups were defined. Further geometric concepts were defined and studied in $[4,5]$. Among these the most important for this paper will be the GS-trapezoid and the affine regular pentagon which we revisit in the third section. In the Euclidean geometry GS-trapezoid is a trapezoid whose lengths of parallel sides are in the golden section ratio and affine regular pentagon is the affine image of a regular pentagon.

Motivated by the example $C(q)$ of pentagonal quasigrops, where $q$ is a solution of (1.11), we prove that we can "find" a GS-quasigroup "inside" any pentagonal quasigroup. More precisely, in a given pentagonal quasigroup $(Q, \cdot)$ we can define a new binary operation $*$ on the set $Q$ by $a * b=(b a \cdot a) a \cdot b$, for $a, b \in Q$. It turns out that $(Q, *)$ is a GS-quasigroup. Furthermore, using that result in the third section we define the concepts of the GS-trapezoid and the affine regular pentagon in pentagonal quasigroups.

In the last section we bring two theorems of the Euclidean plane stated and proved in a pentagonal quasigroup. First is the famous Napoleon-Barlotti theorem (see [1]) in the case $n=5$. The claim of the theorem is:

On the sides of an affine regular $n$-gon regular $n$-gons (all either of the same orientation as the base n-gon or all opposite to it) are constructed. Then the centres of these regular $n$-gons form a regular $n$-gon. 
In order to state and prove it the concept of the regular pentagon and its centre and the concept of the affine regular pentagon will be needed.

The other theorem is a problem proposed in [3]. In the statement and the proof of this theorem the concept of the affine regular pentagon and the concept of the regular decagon, along with its centre, will be needed.

\section{Relation Between pentagonal and GS-Quasigroups}

Firstly we bring a very useful identity that holds in GS-quasigroups and give a characterization of GS-quasigroups involving that identity.

Theorem 2.1. Let $(Q, \cdot)$ be a GS-quasigroup. Then for every $a, b \in Q$ the identity

$$
(a \cdot a b) b=a
$$

holds.

Proof. We have

$$
(a \cdot a b) b \stackrel{(1.1)}{=} a(a a \cdot b) \cdot b \stackrel{(1.12)}{=} a
$$

which proves (2.1).

Theorem 2.2. Let $(Q, \cdot)$ be an IM-quasigroup in which (2.1) holds. Then the identity (1.12) holds in $Q$.

Proof. Since $(Q, \cdot)$ is an IM-quasigroup, besides idempotency (1.1) and mediality (1.2), the elasticity (1.3) holds. Let $a, b, c \in Q$. Then there exists $d \in Q$ such that $a=b d$. We have:

$$
\begin{aligned}
a(a b \cdot c) \cdot c & =(b d \cdot(b d \cdot b) c) c \stackrel{(1.2)}{=}(b(b d \cdot b) \cdot d c) c \stackrel{(1.3)}{=}((b \cdot b d) b \cdot d c) c \\
& (1.2) \\
& =((b \cdot b d) d \cdot b c) c \stackrel{(2.1)}{=}(b \cdot b c) c \stackrel{(2.1)}{=} b .
\end{aligned}
$$

That proves (1.12).

The previous two results combined give the next theorem.

THEOREM 2.3. A quasigroup $(Q, \cdot)$ is a GS-quasigroup if and only if it is an IM-quasigroup in which the identity (2.1) holds.

This theorem is very useful when showing that a certain quasigroup is actually a GS-quasigroup, since (2.1) has less number of variables and multiplications than (1.12).

In a quasigroup $(Q, \cdot)$ operations of left and right division are defined by

$$
a \backslash c=b \Leftrightarrow a b=c \Leftrightarrow c / b=a .
$$

Formula is an expression built up from variables using the operations $\cdot, \backslash$ and /. More precisely:

(1) elements of the set $Q$ (variables) are formulae; 
(2) if $\varphi$ and $\psi$ are formulae, then so are $\varphi \cdot \psi, \varphi \backslash \psi$ and $\varphi / \psi$.

A formula $\varphi$ containing at most two variables gives rise to a new binary operation $Q \times Q \rightarrow Q$, which will also be denoted by $\varphi$.

In [6] the next corollary was proved. We will use it in the proof of the main theorem of the section.

COROLlary 2.4. If $(Q, \cdot)$ is a medial quasigroup, then binary operation defined by the formula $\varphi$ is also medial.

If we choose the model $C\left(q_{1}\right)$ for pentagonal quasigroups, the elements $a$, $b$ and $a b$ of a pentagonal quasigroup $(Q, \cdot)$ form an isosceles triangle $(a, b, a b)$ with angle sizes $36^{\circ}, 108^{\circ}$ and $36^{\circ}$ respectively (so called characteristic triangle). Similarly, if $a$ and $b$ are elements of a GS-quasigroup $(Q, *)$, then the point $a * b$ lies on the line determined by the points $a$ and $b$ and divides the segment $\{a, b\}$ in the golden section ratio.

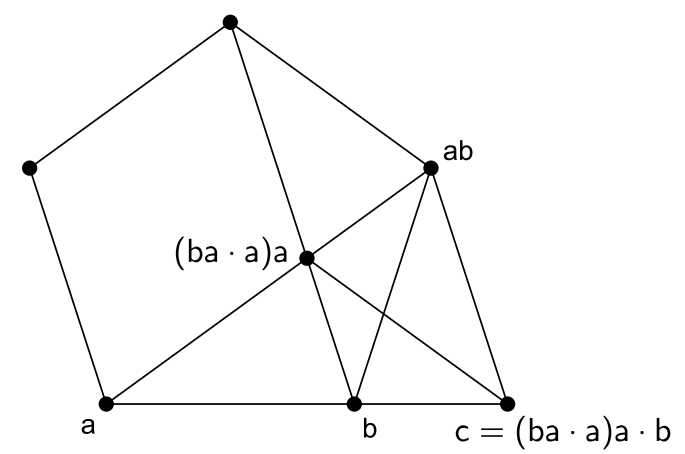

Figure 1. Definition of GS-quasigroup "inside" of pentagonal quasigroup

The next statement is justified by consideration of the geometrical interpretation of a binary operation in a pentagonal quasigroup.

Theorem 2.5. Let $(Q, \cdot)$ be a pentagonal quasigroup and let $*: Q \times Q \rightarrow Q$ be a binary operation defined by

$$
a * b=(b a \cdot a) a \cdot b .
$$

Then $(Q, *)$ is a GS-quasigroup.

Proof. By Theorem 2.3 it is enough to prove that $(Q, *)$ is a quasigroup which satisfies identities of idempotency, mediality and (2.1).

Let $a, b \in Q$ and we observe the equation $a * x=b$. We want to prove that there exists a unique $x \in Q$ which satisfies this equation. Rewriting the 
expression $a * x$ we get:

$$
\begin{aligned}
a * x & =(x a \cdot a) a \cdot x \stackrel{(1.9)}{=}(x a \cdot a) a \cdot(a x \cdot(x a \cdot a) a) \\
& \stackrel{(1.2)}{=}((x a \cdot a) \cdot a x)(a \cdot(x a \cdot a) a) \stackrel{(1.3),(1.8)}{=}((x a \cdot a) \cdot a x)(x a \cdot x) \\
& \stackrel{(1.2)}{=}((x a \cdot a) \cdot x a)(a x \cdot x) \stackrel{(1.5)}{=}(x a \cdot x) a \cdot(a x \cdot x) \\
& \stackrel{(1.4),(1.6)}{=}((x a \cdot x) a \cdot a x) a \stackrel{(1.4),(1.6)}{=}((x a \cdot x) a \cdot a) a \cdot a \\
& \stackrel{(1.8)}{=}(((a x \cdot a) a \cdot a) a \cdot a) a \cdot a .
\end{aligned}
$$

We get the equation

$$
(((a x \cdot a) a \cdot a) a \cdot a) a \cdot a=b .
$$

Since $(Q, \cdot)$ is a quasigroup, dividing by a seven times we get the equivalent equation

$$
a x=c,
$$

where $c \in Q$ and $c=\varphi(a, b)$ for some formula $\varphi$. Now we know that there exists a unique $x \in Q$ such that $a x=c$ holds so we conclude that there exists a unique $x \in Q$ which satisfies

$$
a * x=(((a x \cdot a) a \cdot a) a \cdot a) a \cdot a=b .
$$

Let us now check that $y=(a b \cdot a) a$ satisfies the equation $y * a=b$. We have:

$$
\begin{aligned}
y * a & =(a y \cdot y) y \cdot a=(((a \cdot(a b \cdot a) a) \cdot(a b \cdot a) a) \cdot(a b \cdot a) a) a \\
& \stackrel{(1.2)}{=}((a(a b \cdot a) \cdot((a b \cdot a) a \cdot a)) \cdot(a b \cdot a) a) a \\
& \stackrel{(1.8)}{=}((a(a b \cdot a) \cdot(b a \cdot b)) \cdot(a b \cdot a) a) a \\
& \stackrel{(1.2)}{=}(((a \cdot b a) \cdot(a b \cdot a) b) \cdot(a b \cdot a) a) a \\
& \stackrel{(1.3),(1.2)}{=}((a b \cdot a)(a b \cdot a) \cdot((a b \cdot a) b \cdot a)) a \\
& \stackrel{(1.1),(1.6)}{=}(a b \cdot a) b \cdot a \stackrel{(1.6)}{=} b .
\end{aligned}
$$

Let us assume that there exist $y_{1}, y_{2} \in Q$ such that $y_{1} * a=y_{2} * a=b$. Rewriting that we get

$$
\left(a y_{1} \cdot y_{1}\right) y_{1} \cdot a=\left(a y_{2} \cdot y_{2}\right) y_{2} \cdot a .
$$

Cancelling by $a$ from the right that equation is equivalent to

$$
\left(a y_{1} \cdot y_{1}\right) y_{1}=\left(a y_{2} \cdot y_{2}\right) y_{2} \text {. }
$$

Multiplying by $y_{1} a$ from the left and applying (1.9) we get

$$
a=y_{1} a \cdot\left(a y_{2} \cdot y_{2}\right) y_{2} .
$$


Using (1.9) again it follows

$$
y_{2} a \cdot\left(a y_{2} \cdot y_{2}\right) y_{2}=y_{1} a \cdot\left(a y_{2} \cdot y_{2}\right) y_{2} .
$$

Cancelling by $\left(a y_{2} \cdot y_{2}\right) y_{2}$ from the right we get

$$
y_{2} a=y_{1} a,
$$

which canceling from the right by $a$ finally gives $y_{2}=y_{1}$. We conclude that there is a unique $y \in Q$ such that $y * a=b$ holds and

$$
y=(a b \cdot a) a \text {. }
$$

Hence, $(Q, *)$ is a quasigroup.

We have $a * a=(a a \cdot a) a \cdot a \stackrel{(1.1)}{=} a$ so idempotency holds in $(Q, *)$. By Corollary 2.4 mediality in $(Q, *)$ follows immediately.

It remains to prove

$$
(a *(a * b)) * b=a .
$$

Since the solution of the equation $y * b=a$ is unique, by (2.2) it is enough to prove

$$
a *(a * b)=(b a \cdot b) b .
$$

Since $a * b=(b a \cdot a) a \cdot b$ holds, we get:

$$
\begin{aligned}
a *(a * b) & =((a * b) a \cdot a) a \cdot(a * b)=((a * b) a \cdot a) a \cdot((b a \cdot a) a \cdot b) \\
& \stackrel{(1.2)}{=}(((a * b) a \cdot a) \cdot(b a \cdot a) a) \cdot a b \\
& =((((b a \cdot a) a \cdot b) a \cdot a) \cdot(b a \cdot a) a) \cdot a b \\
& (1.5),(1.3),(1.8) \\
= & (((b a \cdot a) a \cdot b) a \cdot(b a \cdot a) a)(b a \cdot b) \cdot a b \\
& (1.5),(1.3),(1.8) \\
= & (((b a \cdot a) a \cdot b) \cdot(b a \cdot a) a)(b a \cdot b) \cdot(b a \cdot b)) \cdot a b \\
& \stackrel{(1.2),(1.5)}{=}((((b a \cdot b) a \cdot a) \cdot b a)(b a \cdot b) \cdot(b a \cdot b)) \cdot a b \\
& \stackrel{(1.5)}{=}(((b a \cdot b) a \cdot b) a \cdot(b a \cdot b))(b a \cdot b) \cdot a b \\
& \stackrel{(1.6),(1.1)}{=}(a(b a \cdot b) \cdot(b a \cdot b)) \cdot a b \\
& \stackrel{(1.2)}{=}(a(b a \cdot b) \cdot a)(b a \cdot b) b \stackrel{(1.4)}{=}((a \cdot b a) \cdot a b) a \cdot(b a \cdot b) b \\
& \stackrel{(1.3)}{=}((a b \cdot a) \cdot a b) a \cdot(b a \cdot b) b \stackrel{(1.4)}{=}((a b \cdot a) a \cdot(a b \cdot a) b) a \cdot(b a \cdot b) b \\
& \stackrel{(1.5),(1.8),(1.6)}{=}(b a \cdot b) b \cdot(b a \cdot b) b \stackrel{(1.1)}{=}(b a \cdot b) b .
\end{aligned}
$$

Finally, $(Q, *)$ is a GS-quasigroup.

In the previous proof we implicitly proved an identity which will be of a great use in the following sections. We bring it as a corollary. 
Corollary 2.6. Let $(Q, \cdot)$ be a pentagonal quasigroup and $a, b \in Q$. Let $(Q, *)$ be its associated GS-quasigroup, where $a * b=(b a \cdot a) a \cdot b$. Then

$$
a *(a * b)=(b a \cdot b) b
$$

holds.

\section{Affine Regular PEntagons in PEntagonal QUASIGRoups}

In [4] the concept of GS-trapezoid was defined in a GS-quasigroup. That is, according to the example $C\left(\frac{1+\sqrt{5}}{2}\right)$, a trapezoid whose lengths of parallel sides are in the golden section ratio. In [4] it was formulated in the following way.

Definition 3.1. Let $(Q, \cdot)$ be a GS-quasigroup and $a, b, c, d \in Q$. We say that quadrilateral $(a, b, c, d)$ is GS-trapezoid and we denote it by $G S T(a, b, c, d)$ if

$$
a \cdot a b=d \cdot d c
$$

holds (Figure 2).

We notice that a GS-trapezoid in a GS-quasigroup is completely determined by its three vertices. It is obvious that $G S T(a, b, c, d) \Leftrightarrow G S T(d, c, b, a)$ holds.

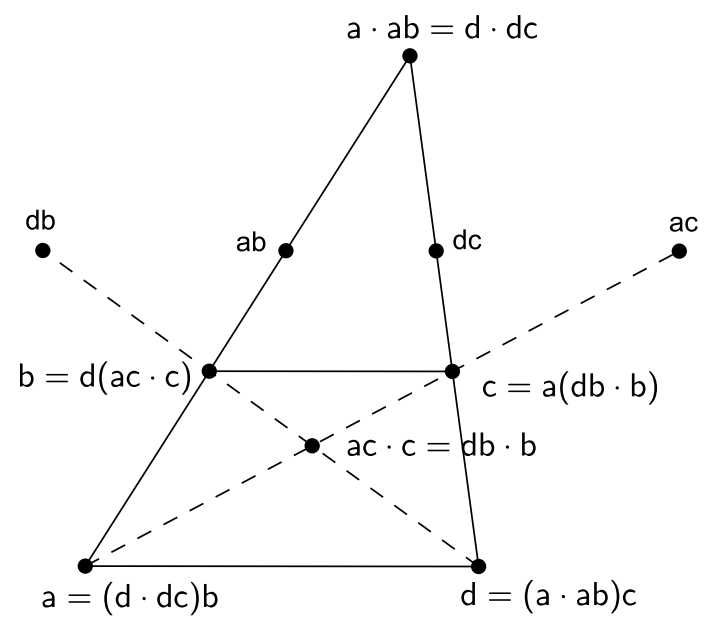

FiguRE 2. GS-trapezoid in GS-quasigroup ([4])

In the already mentioned article the next theorem was proved. 
Theorem 3.2. Let $(Q, \cdot)$ be a GS-quasigroup and $a, b, c, d \in Q$. The relation $G S T(a, b, c, d)$ is equivalent to any of the following four equalities

$$
a=(d \cdot d c) b, b=d(a c \cdot c), c=a(d b \cdot b), d=(a \cdot a b) c .
$$

In the previous section we proved that "inside" of any pentagonal quasigroup we can define a GS-quasigroup. Now we want to define GS-trapezoid in a pentagonal quasigroup.

THEOREM 3.3. Let $(Q, \cdot)$ be a pentagonal quasigroup and $a, b, c, d \in Q$. Let $(Q, *)$ be its associated GS-quasigroup, where $a * b=(b a \cdot a) a \cdot b$. Then

$$
G S T(a, b, c, d) \text { in }(Q, *) \Leftrightarrow d=(c a \cdot b) a \cdot c .
$$

Proof. The relation $G S T(a, b, c, d)$ in $(Q, *)$ is by Theorem 3.2 equivalent to the equality $d=(a *(a * b)) * c$. By Corollary $2.6 a *(a * b)=(b a \cdot b) b$ holds. That implies:

$$
\begin{aligned}
d & =(a *(a * b)) * c=(b a \cdot b) b * c \\
& =(((c \cdot(b a \cdot b) b) \cdot(b a \cdot b) b) \cdot(b a \cdot b) b) c \\
& \stackrel{(1.2),(1.8)}{=}((c(b a \cdot b) \cdot(a b \cdot a)) \cdot(b a \cdot b) b) c \\
& \stackrel{(1.2)}{=}(((c \cdot a b) \cdot(b a \cdot b) a) \cdot(b a \cdot b) b) c \\
& \stackrel{(1.2),(1.6)}{=}((c \cdot a b)(b \cdot a b) \cdot a) c \\
& \stackrel{(1.5)}{=}(c b \cdot a b) a \cdot c \stackrel{(1.5)}{=}(c a \cdot b) a \cdot c .
\end{aligned}
$$

That justifies the next definition of a GS-trapezoid in a pentagonal quasigroup.

DeFinition 3.4. Let $(Q, \cdot)$ be a pentagonal quasigroup and $a, b, c, d \in Q$. We say that quadrilateral $(a, b, c, d)$ is a GS-trapezoid and we denote it by $G S T(a, b, c, d)$ if

holds (Figure 3).

$$
d=(c a \cdot b) a \cdot c
$$

We immediately see that a GS-trapezoid in a pentagonal quasigroup is completely determined by its three vertices.

Affine regular pentagon in the Euclidean plane is the affine image of a regular pentagon. In [5] the notion of the affine regular pentagon in GSquasigroups was defined. In the mentioned paper the next theorem was proved.

Theorem 3.5. Let $(Q, \cdot)$ be a GS-quasigroup and $a, b, c, d, e \in Q$. Any two out of five following relations imply the remaining ones:

$G S T(a, b, c, d), G S T(b, c, d, e), G S T(c, d, e, a), G S T(d, e, a, b), G S T(e, a, b, c)$. 


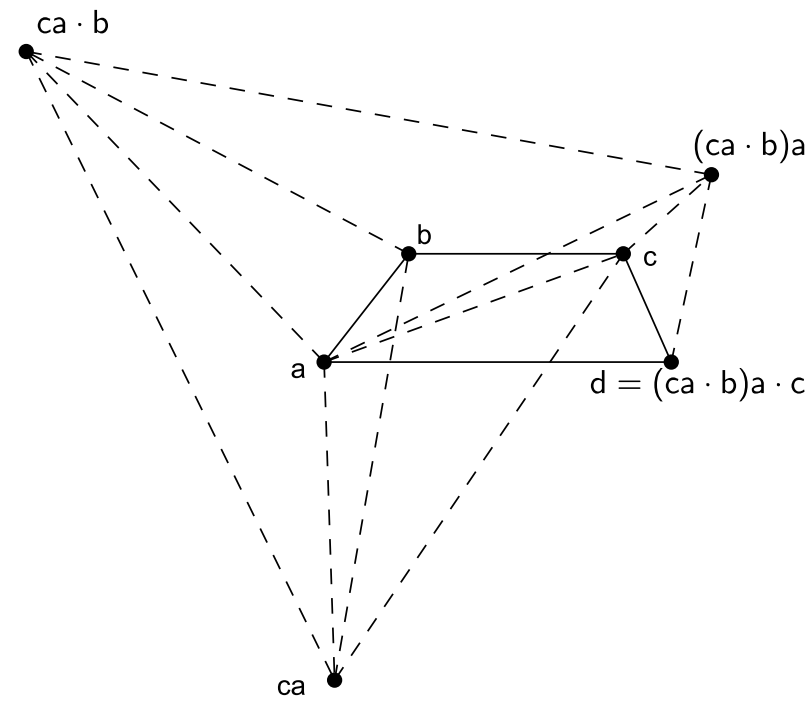

FiguRE 3. Definition of GS-trapezoid in pentagonal quasigroup

That enables the next definition.

Definition 3.6. Let $(Q, \cdot)$ be a GS-quasigroup and $a, b, c, d, e \in Q . W e$ say that pentagon $(a, b, c, d, e)$ is affine regular pentagon and we denote it by $\operatorname{ARP}(a, b, c, d, e)$, if any two (and then all five) out of the following five relations

$G S T(a, b, c, d), G S T(b, c, d, e), G S T(c, d, e, a), G S T(d, e, a, b), G S T(e, a, b, c)$ hold (Figure 4).

It is immediately clear that an affine regular pentagon in GS-quasigroup is uniquely determined by any of its three vertices. We would like to define the affine regular pentagon in the pentagonal quasigroup associated to the GS-quasigroup where that pentagon is already defined.

TheOREm 3.7. Let $(Q, \cdot)$ be a pentagonal quasigroup and $a, b, c, d, e \in Q$. Let $(Q, *)$ be its associated $G S$-quasigroup, where $a * b=(b a \cdot a) a \cdot b$. Then

$$
A R P(a, b, c, d, e) \text { in }(Q, *) \Leftrightarrow d=(c a \cdot b) a \cdot c, e=(a c \cdot b) c \cdot a .
$$

Proof. In $(Q, *)$ we have the equivalence

$$
A R P(a, b, c, d, e) \Leftrightarrow G S T(a, b, c, d), G S T(e, a, b, c) .
$$

By Theorem $3.3 G S T(a, b, c, d) \Leftrightarrow d=(c a \cdot b) a \cdot c$. Since $G S T(e, a, b, c) \Leftrightarrow$ $G S T(c, b, a, e)$ (see [4]) and by Theorem 3.3 we have $G S T(c, b, a, e) \Leftrightarrow e=$ 
$(a c \cdot b) c \cdot a$, we get $G S T(e, a, b, c) \Leftrightarrow e=(a c \cdot b) c \cdot a$. Finally we conclude

$A R P(a, b, c, d, e)$ in $(Q, *) \Leftrightarrow d=(c a \cdot b) a \cdot c, e=(a c \cdot b) c \cdot a$.

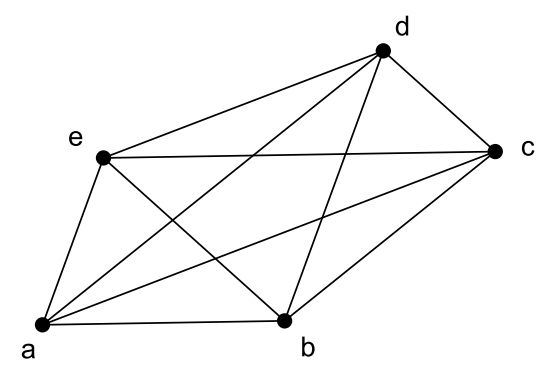

FiguRE 4. Definition of affine regular pentagon in GS-quasigroup

That brings us to the next definition.

DeFinition 3.8. Let $(Q, \cdot)$ be a pentagonal quasigroup and $a, b, c, d, e \in Q$. We say that pentagon $(a, b, c, d, e)$ is affine regular pentagon and we denote it by $\operatorname{ARP}(a, b, c, d, e)$ if

$$
d=(c a \cdot b) a \cdot c, e=(a c \cdot b) c \cdot a
$$

hold (Figure 5).

It is clear that an affine regular pentagon is uniquely determined by its three vertices.

The concept of the regular pentagon in pentagonal quasigroups was defined in [9].

Definition 3.9. We shall say that the points $a, b, c, d, e$ in a pentagonal quasigroup $(Q, \cdot)$ are the vertices of regular pentagon and denote it by $R P(a, b, c, d, e)$ if $a b=c, b c=d$ and $c d=e$ hold.

The next theorem says that any regular pentagon in pentagonal quasigroups is affine regular.

TheOREM 3.10. Let $(Q, \cdot)$ be a pentagonal quasigroup and $a, b, c, d, e \in Q$. The next implication holds

$$
R P(a, b, c, d, e) \Rightarrow A R P(a, b, c, d, e) .
$$




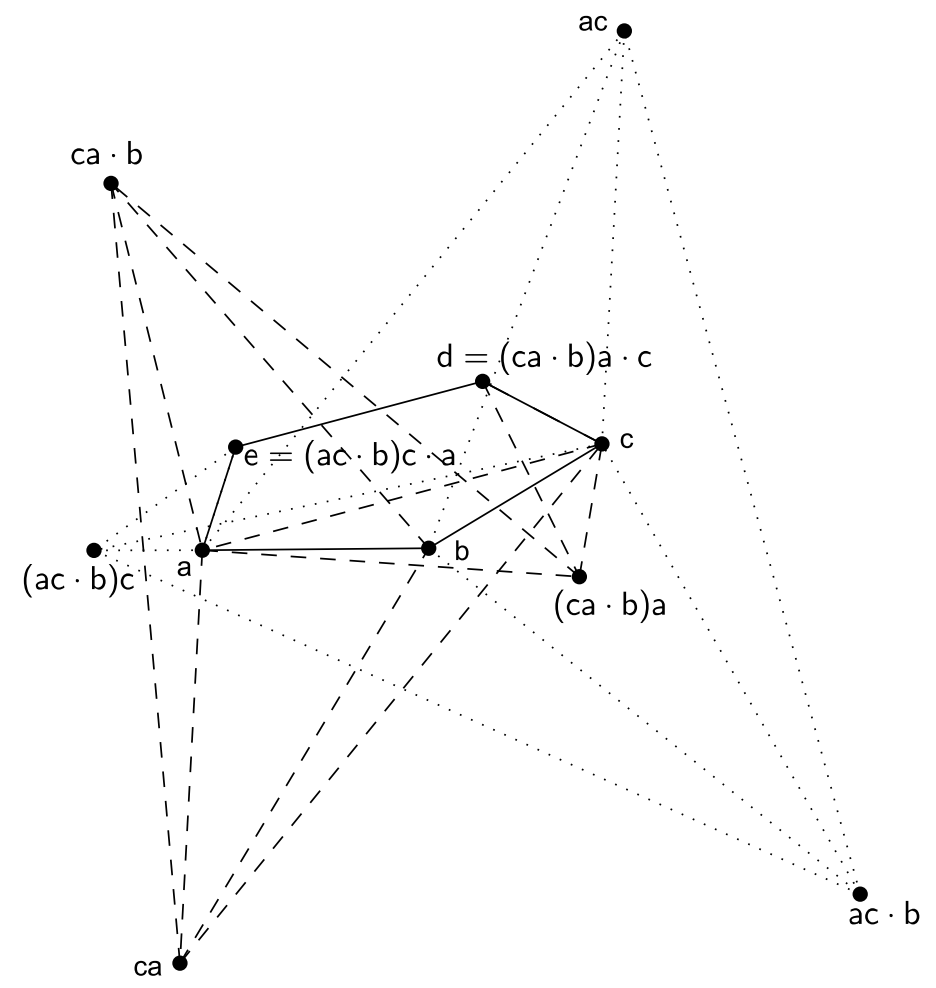

Figure 5. Definition of affine regular pentagon in pentagonal quasigroup

Proof. Because of $R P(a, b, c, d, e)$ we have $c=a b$ and $d=b c=b \cdot a b$ (see [9]). We get

$$
(c a \cdot b) a \cdot c=((a b \cdot a) b \cdot a) \cdot a b \stackrel{(1.6)}{=} b \cdot a b=d .
$$

Similarly, because of $R P(a, b, c, d, e)$ we have $e=(a b \cdot a) b$ (see [9]). We get

$$
\begin{aligned}
(a c \cdot b) c \cdot a & =((a \cdot a b) b \cdot a b) a \stackrel{(1.5)}{=}((a \cdot a b) a \cdot b) a \\
& \stackrel{(1.7)}{=}(a b \cdot b) \cdot a b \stackrel{(1.5)}{=}(a b \cdot a) b=e,
\end{aligned}
$$

which means that $\operatorname{ARP}(a, b, c, d, e)$ holds. 
Along with the concepts of GS-trapezoid and affine regular pentagon, Theorem 2.5 enables us to define all other geometric concepts from GSquasigroups in pentagonal quasigroups. We can say that pentagonal quasigroups "inherit" the entire geometry of GS-quasigroups (see [4,5]) and have their own geometry based on regular pentagons and regular decagons (see [9]).

\section{The Napoleon-Barlotti theorem And similar Results}

In [7] the famous Napoleon-Barlotti theorem in the case $n=3$ was stated and proved in Napoleon's quasigroups. The Napoleon-Barlotti theorem in the case $n=5$ says:

On the sides of an affine regular pentagon regular pentagons are constructed (all on the outer or all on the inner side of that pentagon). Then the centres of these regular pentagons form a regular pentagon.

It is enough to treat the case where all regular pentagons are constructed on the outer side of the affine regular pentagon. In the Figure 6 all regular pentagons are constructed on the outer side of the original affine regular pentagon and that figure is made in the model $C\left(q_{1}\right)$ for pentagonal quasigroups. The case where regular pentagons are constructed on the inner side would correspond to the model $C\left(q_{2}\right)$, so once the theorem is proved in a general pentagonal quasigroup, the both cases are covered.

A minor problem occurs in the statement of the Napoleon-Barlotti theorem in pentagonal quasigroups since the centre of a regular pentagon does not have to exists and if it exists, it does not have to be unique. That problem produces a slightly weaker version of the theorem. The concept of the centre of a regular pentagon was defined in [9].

Definition 4.1. Let $(Q, \cdot)$ be a pentagonal quasigroup and $a, b, c, d, e \in Q$ such that $R P(a, b, c, d, e)$ holds. We say that $o \in Q$ is the centre of the regular pentagon $(a, b, c, d, e)$ if $o a \cdot b=o$ holds.

EXAMPLE 4.2. The smallest non-trivial example of finite pentagonal quasigroup is $\left(Q_{5}, \cdot 1\right)$.

TABLE 1. Pentagonal quasigroup $\left(Q_{5}, \cdot{ }_{1}\right)$

\begin{tabular}{c|ccccc}
$\cdot 1$ & 0 & 1 & 2 & 3 & 4 \\
\hline 0 & 0 & 2 & 4 & 1 & 3 \\
1 & 4 & 1 & 3 & 0 & 2 \\
2 & 3 & 0 & 2 & 4 & 1 \\
3 & 2 & 4 & 1 & 3 & 0 \\
4 & 1 & 3 & 0 & 2 & 4
\end{tabular}

In $\left(Q_{5}, \cdot_{1}\right)$ the statement $R P(0,1,2,3,4)$ holds. The pentagon $(0,1,2,3,4)$ is determined by its vertices 0 and 1 and it has no centre, as shown in [9]. 


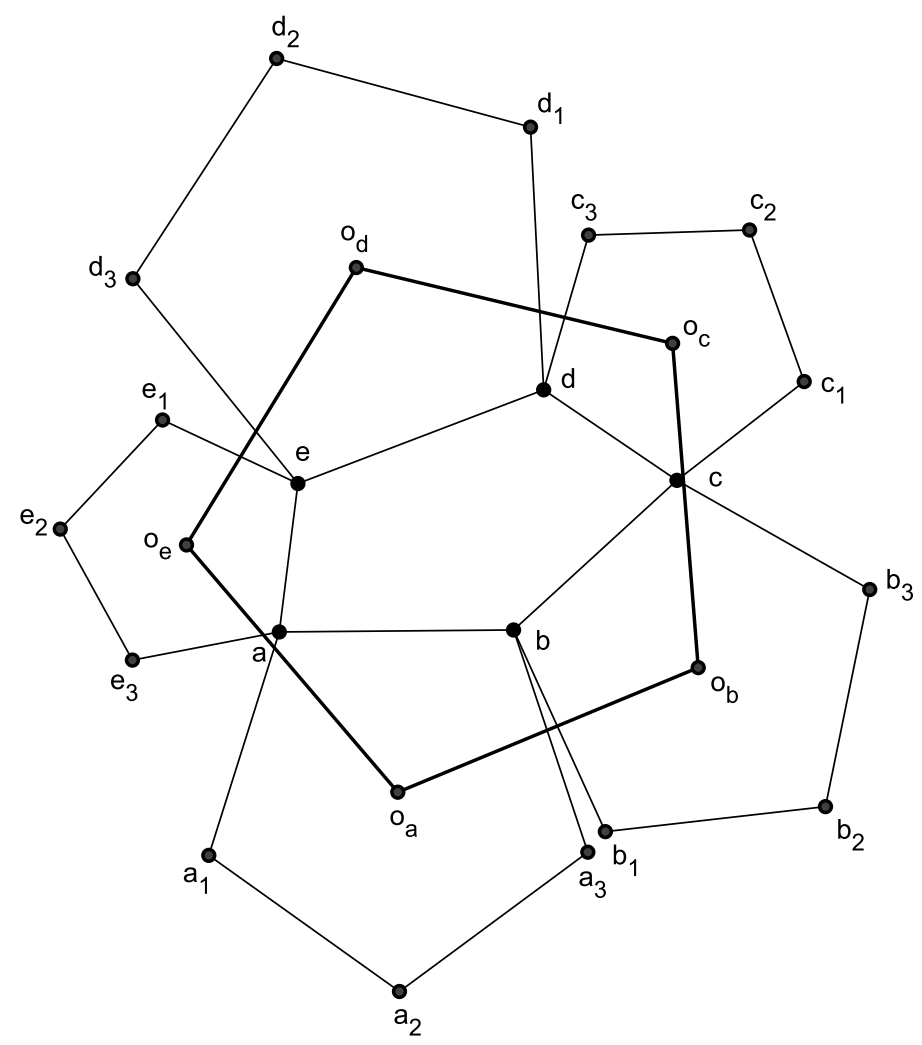

Figure 6. The Napoleon-Barlotti theorem

However, the statement $R P(0,0,0,0,0)$ also holds. The pentagon $(0,0,0,0,0)$ is determined by its vertices 0 and 0 . It is easily checked that every element of $Q_{5}$ is its centre:

$$
\begin{gathered}
00 \cdot{ }_{1} 0=00=0,10 \cdot \cdot_{1} 0=40=1,20 \cdot 10=30=2, \\
30 \cdot \cdot_{1} 0=20=3,40 \cdot{ }_{1} 0=10=4 .
\end{gathered}
$$

EXAMPLE 4.3. The next example of pentagonal quasigroup is $\left(Q_{11}, \cdot_{2}\right)$. In $\left(Q_{11}, \cdot_{2}\right)$ the statement $R P(0,1,4,2,7)$ holds. The pentagon $(0,1,4,2,7)$ is determined by its vertices 0 and 1 . We have:

$$
\begin{gathered}
00 \cdot \cdot_{2} 1=4 \neq 0,10 \cdot{ }_{2} 1=2 \neq 1,20 \cdot \cdot_{2} 1=0 \neq 2,30 \cdot \cdot_{2} 1=9 \neq 3, \\
40 \cdot \cdot_{2} 1=7 \neq 4,50 \cdot{ }_{2} 1=5,60 \cdot \cdot_{2} 1=3 \neq 6,70 \cdot{ }_{2} 1=1 \neq 7, \\
80 \cdot \cdot_{2} 1=10 \neq 8,90 \cdot \cdot_{2} 1=8 \neq 9,100 \cdot \cdot_{2} 1=6 \neq 10 .
\end{gathered}
$$

That means that 5 is the unique centre of the pentagon $(0,1,4,2,7)$. 
TABLE 2. Pentagonal quasigroup $\left(Q_{11}, \cdot 2\right)$

\begin{tabular}{c|ccccccccccc}
$\cdot 2$ & 0 & 1 & 2 & 3 & 4 & 5 & 6 & 7 & 8 & 9 & 10 \\
\hline 0 & 0 & 4 & 8 & 1 & 5 & 9 & 2 & 6 & 10 & 3 & 7 \\
1 & 8 & 1 & 5 & 9 & 2 & 6 & 10 & 3 & 7 & 0 & 4 \\
2 & 5 & 9 & 2 & 6 & 10 & 3 & 7 & 0 & 4 & 8 & 1 \\
3 & 2 & 6 & 10 & 3 & 7 & 0 & 4 & 8 & 1 & 5 & 9 \\
4 & 10 & 3 & 7 & 0 & 4 & 8 & 1 & 5 & 9 & 2 & 6 \\
5 & 7 & 0 & 4 & 8 & 1 & 5 & 9 & 2 & 6 & 10 & 3 \\
6 & 4 & 8 & 1 & 5 & 9 & 2 & 6 & 10 & 3 & 7 & 0 \\
7 & 1 & 5 & 9 & 2 & 6 & 10 & 3 & 7 & 0 & 4 & 8 \\
8 & 9 & 2 & 6 & 10 & 3 & 7 & 0 & 4 & 8 & 1 & 5 \\
9 & 6 & 10 & 3 & 7 & 0 & 4 & 8 & 1 & 5 & 9 & 2 \\
10 & 3 & 7 & 0 & 4 & 8 & 1 & 5 & 9 & 2 & 6 & 10
\end{tabular}

EXAMPLE 4.4. The direct product of the pentagonal quasigroups $\left(Q_{5}, \cdot_{1}\right)$ and $\left(Q_{11}, \cdot_{2}\right)$ is the pentagonal quasigroup $\left(Q_{55}, \cdot\right)$. For $\left(a_{1}, a_{2}\right),\left(b_{1}, b_{2}\right) \in Q_{55}$ we have

$$
\left(a_{1}, a_{2}\right) \cdot\left(b_{1}, b_{2}\right)=\left(a_{1} \cdot{ }_{1} b_{1}, a_{2} \cdot{ }_{2} b_{2}\right) .
$$

From the previous two examples we see that $R P((0,0),(0,1),(0,4),(0,2)$, $(0,7))$ holds. The pentagon $((0,0),(0,1),(0,4),(0,2),(0,7))$ is determined by its vertices $(0,0)$ and $(0,1)$. From

$$
\begin{aligned}
& (0,5)(0,0) \cdot(0,1)=(0,5), \\
& (1,5)(0,0) \cdot(0,1)=(1,5), \\
& (2,5)(0,0) \cdot(0,1)=(2,5), \\
& (3,5)(0,0) \cdot(0,1)=(3,5), \\
& (4,5)(0,0) \cdot(0,1)=(4,5),
\end{aligned}
$$

we conclude that $(0,5),(1,5),(2,5),(3,5),(4,5)$ are 5 distinct centres of the pentagon $((0,0),(0,1),(0,4),(0,2),(0,7))$.

Here we bring the version of the Napoleon-Barlotti theorem in pentagonal quasigroups.

TheOREM 4.5. Let $(Q, \cdot)$ be a pentagonal quasigroup. Let $A R P(a, b, c, d, e)$, $R P\left(b, a, a_{1}, a_{2}, a_{3}\right), R P\left(c, b, b_{1}, b_{2}, b_{3}\right), R P\left(d, c, c_{1}, c_{2}, c_{3}\right), R P\left(e, d, d_{1}, d_{2}, d_{3}\right)$, $R P\left(a, e, e_{1}, e_{2}, e_{3}\right)$ hold and let $o_{a}$ be the centre of $\left(b, a, a_{1}, a_{2}, a_{3}\right)$ and $o_{b}$ be the centre of $\left(c, b, b_{1}, b_{2}, b_{3}\right)$. If $R P\left(o_{a}, o_{b}, o_{c}, o_{d}, o_{e}\right)$ holds, then $o_{c}, o_{d}$ and $o_{e}$ are the centres of the regular pentagons $\left(d, c, c_{1}, c_{2}, c_{3}\right),\left(e, d, d_{1}, d_{2}, d_{3}\right)$ and $\left(a, e, e_{1}, e_{2}, e_{3}\right)$, respectively. 
Proof. Since $o_{a}$ and $o_{b}$ are centres of the regular pentagons $\left(b, a, a_{1}, a_{2}\right.$, $\left.a_{3}\right)$ and $\left(c, b, b_{1}, b_{2}, b_{3}\right)$, respectively, we have

$$
\begin{aligned}
& o_{a} b \cdot a=o_{a}, \\
& o_{b} c \cdot b=o_{b} .
\end{aligned}
$$

Multiplying the equalities (4.1) and (4.2) and using (1.2) we get:

$$
\begin{gathered}
\left(o_{a} b \cdot a\right)\left(o_{b} c \cdot b\right)=o_{a} o_{b}, \\
\left(o_{a} b \cdot o_{b} c\right) \cdot a b=o_{a} o_{b}, \\
\left(o_{a} o_{b} \cdot b c\right) \cdot a b=o_{a} o_{b} .
\end{gathered}
$$

Because of $R P\left(o_{a}, o_{b}, o_{c}, o_{d}, o_{e}\right)$, we have $o_{c}=o_{a} o_{b}$, and we want to prove that $o_{c}$ is the centre of the regular pentagon $\left(d, c, c_{1}, c_{2}, c_{3}\right)$. Hence, we have

$$
o_{c}=\left(o_{c} \cdot b c\right) \cdot a b,
$$

and it is enough to prove

$$
o_{c} d \cdot c=o_{c} \text {. }
$$

Since $\operatorname{ARP}(a, b, c, d, e)$ holds, by Theorem 3.7 or by Definition 3.8, we have $d=(c a \cdot b) a \cdot c$. Hence, we get successively:

$$
\begin{aligned}
o_{c} d \cdot c & =o_{c}((c a \cdot b) a \cdot c) \cdot c \stackrel{(4.3)}{=}\left(\left(o_{c} \cdot b c\right) \cdot a b\right)((c a \cdot b) a \cdot c) \cdot c \\
& \stackrel{(1.2)}{=}\left(\left(o_{c} \cdot b c\right) \cdot(c a \cdot b) a\right)(a b \cdot c) \cdot c \stackrel{(1.2)}{=}\left(o_{c}(c a \cdot b) \cdot(b c \cdot a)\right)(a b \cdot c) \cdot c \\
& \stackrel{(1.2)}{=}\left(\left(o_{c}(c a \cdot b) \cdot a b\right) \cdot(b c \cdot a) c\right) c \stackrel{(1.2)}{=}\left(\left(o_{c} a \cdot(c a \cdot b) b\right) \cdot(b c \cdot a) c\right) c \\
& \stackrel{(1.2)}{=}\left(o_{c} a \cdot(b c \cdot a)\right)((c a \cdot b) b \cdot c) \cdot c \stackrel{(1.5)}{=}\left(\left(o_{c} \cdot b c\right) a \cdot((c a \cdot b) b \cdot c)\right) c \\
& \stackrel{(1.2)}{=}\left(\left(\left(o_{c} \cdot b c\right) \cdot(c a \cdot b) b\right) \cdot a c\right) c \stackrel{(1.2)}{=}\left(\left(o_{c}(c a \cdot b) \cdot(b c \cdot b)\right) \cdot a c\right) c \\
& \stackrel{(1.3)}{=}\left(\left(o_{c}(c a \cdot b) \cdot(b \cdot c b)\right) \cdot a c\right) c \stackrel{(1.2),(1.5)}{=}\left(\left(o_{c} b \cdot(c a \cdot c) b\right) \cdot a c\right) c \\
& \stackrel{(1.2)}{=}\left(o_{c} b \cdot a\right)((c a \cdot c) b \cdot c) \cdot c \stackrel{(1.7)}{=}\left(o_{c} b \cdot a\right)(a b \cdot a) \cdot c \\
& \stackrel{(1.5)}{=}\left(o_{c} b \cdot a b\right) a \cdot c \stackrel{(1.5)}{=}\left(o_{c} a \cdot b\right) a \cdot c \\
& \stackrel{(1.6)}{=}\left(o_{c} a \cdot b\right) a \cdot((b c \cdot b) c \cdot b) \stackrel{(1.2)}{=}\left(\left(o_{c} a \cdot b\right) \cdot(b c \cdot b) c\right) \cdot a b \\
& \stackrel{(1.2)}{=}\left(\left(o_{c} a \cdot(b c \cdot b)\right) \cdot b c\right) \cdot a b \stackrel{(1.2)}{=}\left(\left(\left(o_{c} \cdot b c\right) \cdot a b\right) \cdot b c\right) \cdot a b \\
& \stackrel{(4.3)}{=}\left(o_{c} \cdot b c\right) \cdot a b \stackrel{(4.3)}{=} o_{c}, \quad
\end{aligned}
$$

exactly what we wanted to show. Hence, $o_{c}$ is the centre of the regular pentagon $\left(d, c, c_{1}, c_{2}, c_{3}\right)$.

It can be shown analogously that $o_{d}$ is the centre of the regular pentagon $\left(e, d, d_{1}, d_{2}, d_{3}\right)$ and that $o_{e}$ is the centre of the regular pentagon $\left(a, e, e_{1}, e_{2}, e_{3}\right)$. 
At the end we bring another interesting theorem of the Euclidean plane in pentagonal quasigroups. In [3] the next theorem was stated as a problem:

Let $A B C D E$ be an affine regular pentagon and $D^{\prime}$ and $E^{\prime}$ vertices of the regular pentagons on the sides $\overline{B A}$ and $\overline{C B}$ opposite to that sides, respectively. Let $B^{\prime \prime}$ be the vertex of the regular pentagon on the side $\overline{D^{\prime} E^{\prime}}$ opposite to that side. Then $B^{\prime \prime}=C D \cap E A$.

In order to state and prove that theorem in pentagonal quasigroups we can firstly notice that the vertex of the regular pentagon on the side $\overline{B A}$ opposite to that side is actually the fourth vertex of that regular pentagon and that is exactly the centre of the regular decagon on $\overline{B A}$. We also have to determine what is the intersection of the mentioned lines $C D$ and $E A$. Since $A E D C$ is a GS-trapezoid, that intersection can be reached using only golden section ratio on the line $E A$ or on the line $C D$ (see Figure 2 and [4]).

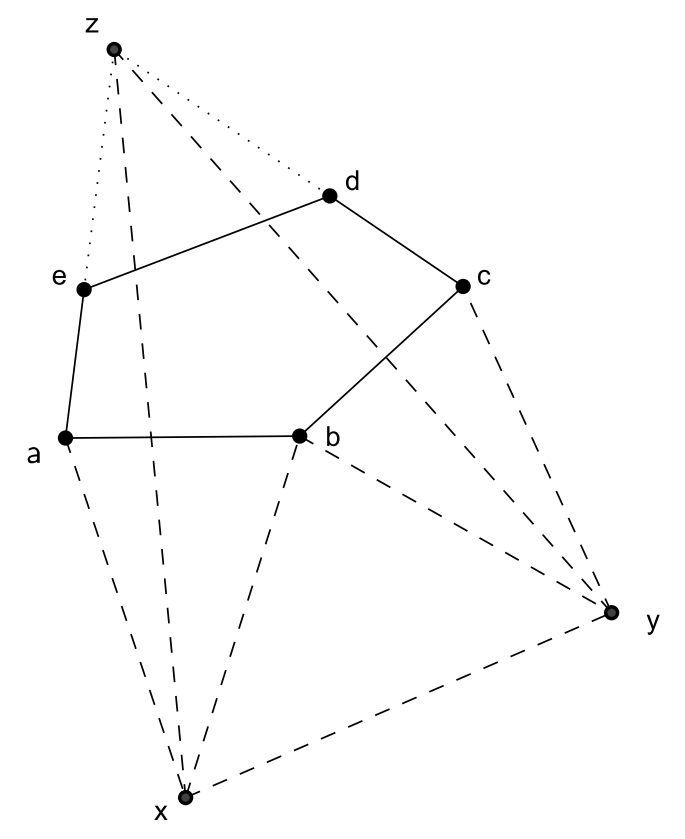

FIGURE 7. The geometrical presentation of Theorem 4.6

TheOREM 4.6. Let $(Q, \cdot)$ be a pentagonal quasigroup and let $(Q, *)$ be its associated GS-quasigroup. Let $\operatorname{ARP}(a, b, c, d, e)$ hold and let $x$ and $y$ be the fourth vertices of the regular pentagons determined by segments $\{b, a\}$ and $\{c, b\}$, respectively. If $z=a *(a * e)$, then $z$ is the fourth vertex of the regular pentagon determined by segment $\{x, y\}$. 
Proof. Since $G S T(a, e, d, c)$ holds, $z=a *(a * e)=c *(c * d)$. By Corollary 2.6 we have $z=a *(a * e)=(e a \cdot e) e$.

Since $x$ is the vertex of the regular pentagon determined by segment $\{b, a\}$, we have $x=a b \cdot a$ (see [9]). Analogously we have $y=b c \cdot b$.

In order to prove the statement of the theorem it is enough to prove $z=y x \cdot y$. Since $\operatorname{ARP}(a, b, c, d, e)$ holds, by Theorem 3.7 or by Definition 3.8 we have $e=(a c \cdot b) c \cdot a$. We get successively:

$$
\begin{aligned}
z & =(e a \cdot e) e=(((a c \cdot b) c \cdot a) a \cdot((a c \cdot b) c \cdot a)) e \\
& \stackrel{(1.5)}{=}(((a c \cdot b) c \cdot a) \cdot(a c \cdot b) c) a \cdot e \stackrel{(1.2)}{=}(((a c \cdot b) c \cdot(a c \cdot b)) \cdot a c) a \cdot e \\
& \stackrel{(1.2)}{=}((((a c \cdot b) \cdot a c) \cdot c b) \cdot a c) a \cdot e \stackrel{(1.7)}{=}((b \cdot c b) b \cdot a) \cdot((a c \cdot b) c \cdot a) \\
& \stackrel{(1.3),(1.5)}{=}((b c \cdot b) b \cdot(a c \cdot b) c) a \stackrel{(1.2)}{=}((b c \cdot b)(a c \cdot b) \cdot b c) a \\
& \stackrel{(1.5),(1.5)}{=}((b a \cdot c) b \cdot b c) a \stackrel{(1.5)}{=}(((b a \cdot b) \cdot c b) \cdot b c) a \\
& \stackrel{(1.2)}{=}((b a \cdot b) b \cdot(c b \cdot c)) a \stackrel{(1.8),(1.6)}{=}(((a b \cdot a) a \cdot a)((a b \cdot a) b \cdot a) \cdot(c b \cdot c)) a \\
& \stackrel{(1.5)}{=}(((a b \cdot a) a \cdot(a b \cdot a) b) a \cdot(c b \cdot c)) a \stackrel{(1.4)}{=}(((a b \cdot a) \cdot a b) a \cdot(c b \cdot c)) a \\
& \stackrel{(1.5)}{=}(((a b \cdot a) a \cdot(a b \cdot a)) \cdot(c b \cdot c)) a \stackrel{(1.2)}{=}(((a b \cdot a) a \cdot c b) \cdot(a b \cdot a) c) a \\
& \stackrel{(1.2)}{=}(((a b \cdot a) c \cdot a b) \cdot(a b \cdot a) c) a \stackrel{(1.5)}{=}((a b \cdot a) c \cdot a b) a \cdot((a b \cdot a) c \cdot a) \\
& \stackrel{(1.5)}{=}((a b \cdot a) c \cdot a)(a b \cdot a) \cdot((a b \cdot a) c \cdot a) \stackrel{(1.7),(1.7)}{=}(b c \cdot b)(a b \cdot a) \cdot(b c \cdot b) \\
= & y x \cdot y,
\end{aligned}
$$

which is exactly what we needed to prove.

\section{REFERENCES}

[1] A. Barlotti, Intorno ad una generazione di un noto teorema relativo al triangolo, Boll. Uni. Math. Ital. 7 (1952), 182-185.

[2] M. Bombardelli and V. Volenec, Vectors and transfers in hexagonal quasigroup, Glas. Mat. Ser. III 42 (62) (2007), 363-373.

[3] E. Kitchen, Problem 1979, Crux Math. 20 (1994), 226.

[4] Z. Kolar and V. Volenec, GS-trapezoids in GS-quasigroups, Math. Commun. 7 (2002), 143-158.

[5] Z. Kolar-Begović and V. Volenec, Affine regular pentagons in GS-quasigroups, Quasigroups Related Systems 12 (2004), 103-112.

[6] V. Krčadinac and V. Volenec, A note on medial quasigroups, Math. Commun. 11 (2006), 83-85.

[7] V. Krčadinac, Napoleon's quasigroups, Math. Slovaca 61 (2011), 885-894.

[8] S. Vidak, Pentagonal quasigroups, Quasigroups Related Systems 22 (2014), 147-158.

[9] S. Vidak, Geometry of pentagonal quasigroups, Publ. Inst. Math. (Beograd) (N.S.) 99(113) (2016), 109-120.

[10] V. Volenec, Geometry of medial quasigroups, Rad Jugoslav. Akad. Znan. Umjet. 5 (1986), 79-91. 
[11] V. Volenec, GS-quasigroups, Časopis Pěst. Mat. 115 (1990), 307-318.

[12] V. Volenec, Geometry of IM-quasigroups, Rad Hrvatske Akad. Znan. Umjet. 10 (1991), 139-146.

S. Vidak

Department of Mathematics

University of Zagreb

10000 Zagreb

Croatia

E-mail: svidak@math.hr

Received: 21.9.2015.

Revised: 16.3.2016. 